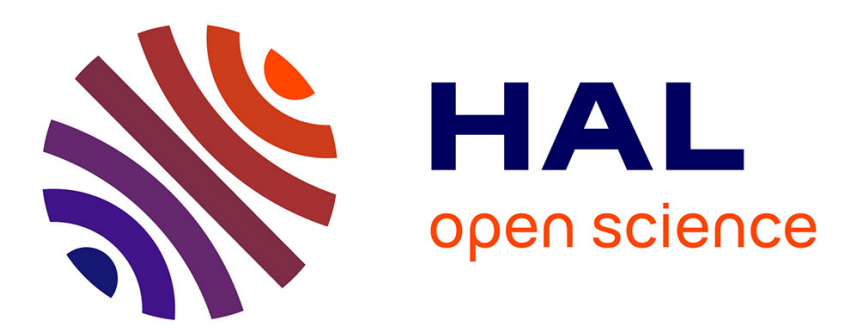

\title{
Electrolyte-Gated Field Effect Transistor to Probe the Surface Defects and Morphology in Films of Thick CdSe Colloidal Nanoplatelets
}

Emmanuel Lhuillier, Silvia Pedetti, Sandrine Ithurria, Hadrien Heuclin, Brice Nadal, Adrien Robin, Gilles Patriarche, Nicolas Lequeux, Benoit Dubertret

\section{To cite this version:}

Emmanuel Lhuillier, Silvia Pedetti, Sandrine Ithurria, Hadrien Heuclin, Brice Nadal, et al.. Electrolyte-Gated Field Effect Transistor to Probe the Surface Defects and Morphology in Films of Thick CdSe Colloidal Nanoplatelets. ACS Nano, 2014, 8 (4), pp.3813 - 3820. 10.1021/nn500538n . hal-01438569

\section{HAL Id: hal-01438569 https://hal.science/hal-01438569}

Submitted on 25 Aug 2020

HAL is a multi-disciplinary open access archive for the deposit and dissemination of scientific research documents, whether they are published or not. The documents may come from teaching and research institutions in France or abroad, or from public or private research centers.
L'archive ouverte pluridisciplinaire HAL, est destinée au dépôt et à la diffusion de documents scientifiques de niveau recherche, publiés ou non, émanant des établissements d'enseignement et de recherche français ou étrangers, des laboratoires publics ou privés. 


\title{
Electrolyte Gated Field Effect Transistor to Probe the Surface Defects and Morphology in Films of Thick CdSe Colloidal Nanoplatelets
}

\author{
Emmanuel Lhuillier ${ }^{1}$, Silvia Pedetti ${ }^{1,2}$, Sandrine Ithurria ${ }^{2}$, Hadrien Heuclin ${ }^{1}$, Brice Nadal ${ }^{1}$, Adrien \\ Robin $^{2}$, Gilles Patriarche ${ }^{3}$, Nicolas Lequeux ${ }^{2}$ and Benoit Dubertret ${ }^{2 *}$ \\ ${ }^{1}$ Solarwell, 10 rue Vauquelin, Paris, France \\ ${ }^{2} \mathrm{LPEM}, \mathrm{ESPCI} 10$ rue Vauquelin, Paris, France
}

${ }^{3}$ Laboratoire de Photonique et de Nanostructures, LPN/UPR20-CNRS Route de Nozay, 91460

Marcoussis, France.

\begin{abstract}
The optical and optoelectronic properties of colloidal quantum dots strongly depend on the passivation of their surface. Surface states are however difficult to quantify using optical spectroscopy and techniques based on back gated field effect transistors are limited in the range of carriers density that can be probed, usually significantly below one charge carrier per particle. Here we show that electrolyte gating can be used to quantitatively analyze the increase of defects in a population of nanoparticles with increasing surface irregularities. We illustrate this method using CdSe nanoplatelets that are grown in their thickness using low temperature layer-by-layer method. Spectroscopic analysis of the samples confirm that the nanoplatelet thickness is controlled, on average, with atomic precision, but structural analysis with transmission electron microscopy shows that the number of surface defects increases with the nanoplatelet thickness. The amount of charge defects is probed quantitatively using electrolyte gated field effect transistor (EFET). We observe that the threshold voltage of the EFET increases with the NPLs thickness, in agreement with the structural analysis. All samples displayed n-type conduction with strong current modulation (subthreshold swing slope of $100 \mathrm{mV} /$ decade and on/off ratio close to $10^{7}$ ). We also point out that an efficient electrolyte gating of the film requires a fine control of the nanoparticle film morphology.
\end{abstract}

KEYWORDS: Nanoplatelets, core/shell, heterostructure, electrolyte gating, transistor, stoichiometry defects.

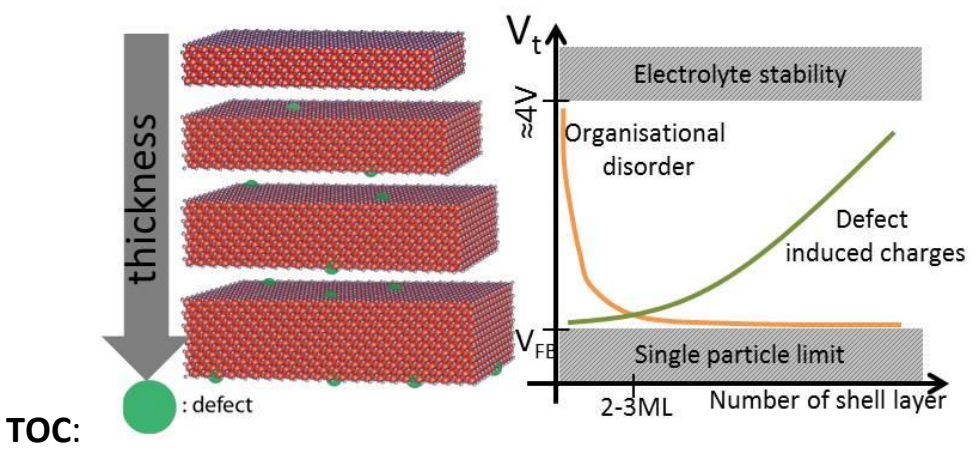


Thanks to twenty years of intense research, great progresses have been achieved in the control of the shape and the dimensionality ${ }^{1}$ of colloidal nanoparticles. Amongst the newly developed shapes, 2D colloidal nanostructures are particularly appealing due to the ability to control the thickness in the confined direction at the atomic scale. ${ }^{2,3}$ Compared to spherical objects this leads to exceptionally narrow excitonic features with a homogenous broadening ${ }^{4}$ close to the thermal energy, and a fast excitonic lifetime. ${ }^{5}$ 2D nanoplatelets (NPLs) can now be synthetized with most cadmium chalchogenides materials $\left(\mathrm{CdS}^{6}, \mathrm{CdSe}^{7}, \mathrm{CdTe}^{8}\right)$ with both zinc blende ${ }^{2}$ and wurtzite ${ }^{9}$ structure. Furthermore, 2D core/shell nanoplatelets have recently be obtained. Since these are fragile objects, low temperature strategies have been developed based either on one pot ${ }^{10}$ or layer-by-layer ${ }^{11}$ procedures.

For practical use in an optoelectronic device ${ }^{12}$ the nanoparticles need to be strongly electronically coupled. The enhancement of the interparticle wavefunction overlap is typically achieved by shortening the capping ligand length. Nevertheless this process affects the particle surface and tends to introduce some surface charges. ${ }^{13}$ Field effect transistor (FET) has appeared as the most versatile tools to characterize the transport properties and carrier density of colloidal quantum dots (CQD) film. In spite of this success, the carrier modulation achievable by a back gated FET is typically below $10^{13} \mathrm{~cm}^{-2}$ corresponding to a density around $2 \times 10^{18} \mathrm{~cm}^{-3}$ for a $50 \mathrm{~nm}$ thick film..$^{14,15}$ To reach higher carrier density ( 1 to 10 carriers per QD), electrolyte gating is necessary. ${ }^{16-18}$ Here we use electrolyte gating to probe the large stoichiometry defects induced during the low temperature growth of a CdSe shell on CdSe core.

In a recent paper we have demonstrated that $\mathrm{CdSe} / \mathrm{CdS}$ nanoplatelets can be used as the canal of a polymer electrolyte gated field effect transistor ${ }^{19}$ with exceptional properties (on/off ratio up to $10^{9}$, subthreshold swing as low as $80 \mathrm{mV} /$ decade and low threshold voltage $<1 \mathrm{~V}$ ). The growth of the $\mathrm{CdS}$ shell seemed to play a key role in the charging process that we further investigate here. We use FET as a probe to investigate the defects due to the nanoparticles' surface and to the morphology of the NPLs' film. We demonstrate that electrolyte gating is an efficient method to probe the charge related to the surface defects, which can be difficultly be addressed in a quantitative way by other methods such as optical spectroscopy. We apply this electrolyte gating to $\mathrm{CdSe} / \mathrm{CdSe}$ semiconductor homostructures with a 2D shape. Their optical properties are investigated in order to understand their band structure and confirm the delocalization of both carriers over the whole structure. Finally we also discuss the gating efficiency as a function of the nanoparticles organization.

\section{RESULTS AND DISCUSSION}

\section{Material and Band Structure}

Recently $\mathrm{Nag}^{20}$ et al proposed the use of $\mathrm{Se}^{2-}$ as an atomic passivation for CdSe QDs. Here we combine this surface ligand with the c-ALD (colloidal atomic layer deposition) procedure developed Ithurria et $a l^{11}$ to achieve the growth of a CdSe shell around the CdSe NPLs. Our procedure is based on the in situ formation of the $\mathrm{Se}^{2-}$ species in a mixture of polar solvents (ethanol and $\mathrm{N}$-methyl formamide) under inert atmosphere. ${ }^{21}$ 

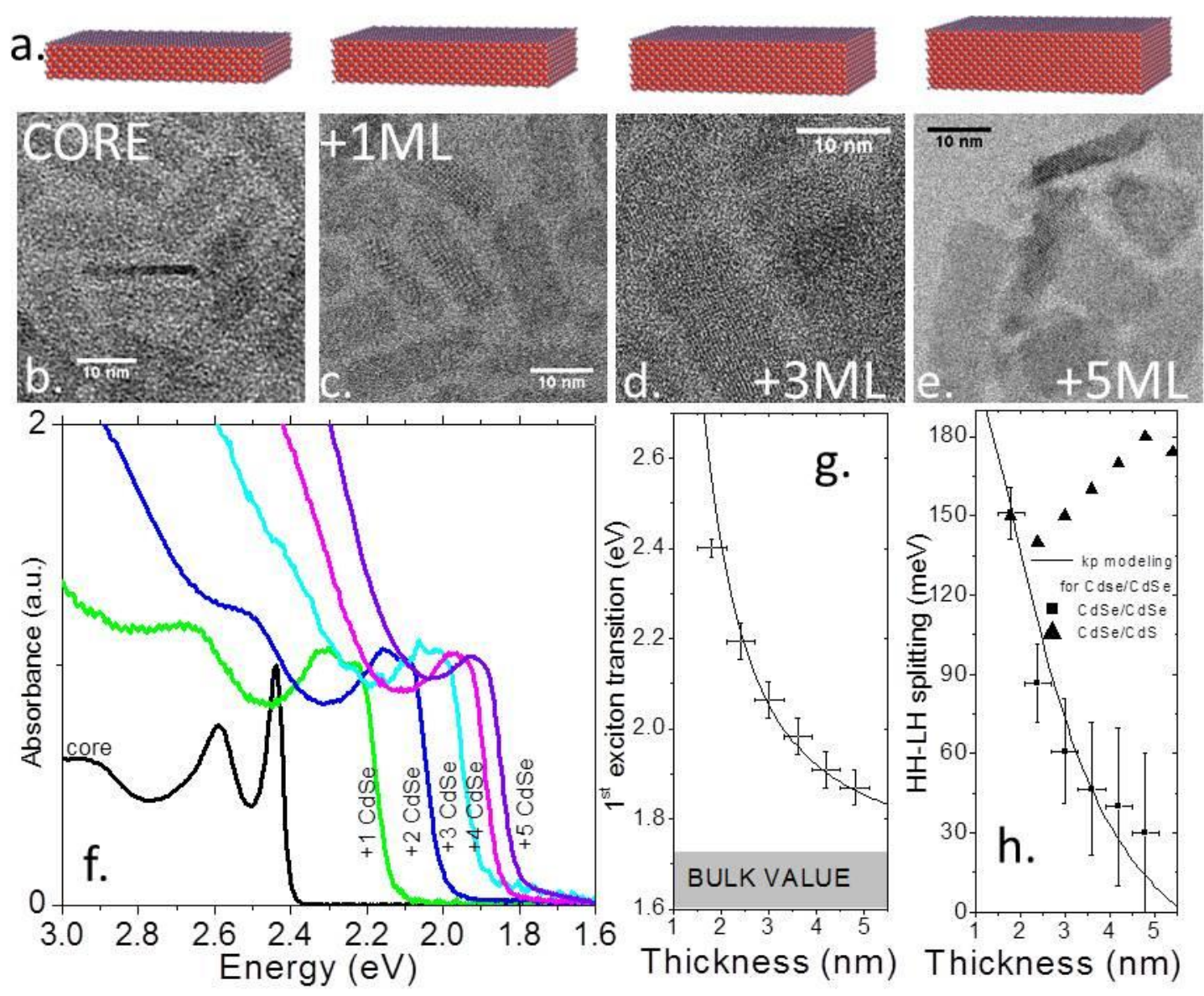

Figure 1 : (a) Scheme of CdSe nanoplatelets with an increasing thickness. TEM image of CdSe NPLs core (b), CdSe/CdSe NPLs with 1 (c), 3 (d) and 5 (e) monolayer(s) on each side. (f): Absorption spectra of CdSe/CdSe NPLs of different thicknesses. Here we assume that for each monolayer the platelets thickness rise by one half lattice parameter on each side, meaning $0.61 \mathrm{~nm}$. The inset is the band structure of CdSe. (g): Energy of the first exciton as a function of the NPLs' thickness. The line results from a k.p modeling of the CdSe/CdSe NPL. (h): Energy of the heavy hole - light hole splitting as a function of the NPLs' thickness. The line results from a $k \cdot p$ modeling of the CdSe/CdSe NPL. The triangles are the values obtained when a CdS shell (instead of a CdSe shell) is grown on a CdSe NPL core.

During the layer-by-layer process, the NPLs thickness increases by two CdSe monolayers (one on each side of the NPL, equivalent to a lattice parameter) at each cycle of anion/cation deposition. This growth results in a gradual red shift of the NPL's exciton transitions combined with a reducing energy splitting between the heavy hole-electron ( $\mathrm{HL}$ ) and light hole-electron (LH) transitions. Both effects can be monitored on Figure 1 (f). The latter behavior is a significant deviation from the $\mathrm{CdSe} / \mathrm{CdS}$ core/shell nanoplatelets for which the HH-LH splitting remains constant while the shell is grown, (see Figure $1(\mathrm{~h})$ ). High resolution transmission electron microscopy (TEM) confirms that the material is epitaxially grown on the CdSe core (see Figure $1(b)$ to (e) and Figure S2). The initial core structure is zinc blende and remains so through the deposition, as confirmed by X-Ray Diffraction (XRD) data (Figure S3). As the NPL thickness increases, the TEM images also show that the NPL surface becomes more and more irregular with potentially an increasing amount of stoichiometry defects (see Figure 1 
(d) and Figure S2). The strong irregularity of the NPLs surfaces challenges the idea that NPLs grow, on average, one CdSe layer at a time at each cycle.

We use $k \cdot p$ simulation ${ }^{5}$ to confirm that the shell growth is effectively acting as a layer by layer procedure. Based on Pidgeon-Brown hamiltonian ${ }^{22}$ the dispersion of the heavy hole is given by $E_{h h}=-\left(\gamma_{1}-2 \gamma\right) E_{K}$ while the dispersion of the electron, light hole and spin orbit bands are given by

$$
\left(E-E_{G}-\alpha E_{K}\right)\left[\left(E+\gamma_{1} E_{K}+\Delta\right)\left(E+\left(\gamma_{1}+2 \gamma\right) E_{K}\right)-8 \gamma^{2} E_{K}^{2}\right]-E_{p} E_{K}\left(E+\frac{2 \Delta}{3}\right)-E_{p}\left(\gamma_{1}-2 \gamma\right) E_{K}^{2}=0
$$

In the last two expressions, $E_{G}$ is the bulk band gap energy (1.66eV), $E_{p}$ the Kane energy $(16.5 \mathrm{eV})$, $\Delta$ the spin orbit energy $(0.39 \mathrm{eV}), \gamma_{1}(-0.18)$ and $\gamma(-0.65)$ are the two Luttinger parameters and finally $\alpha(-1.54)$ is a non-parabolic coefficient. The values of the parameters are the same as given in reference 5 . The kinetic energy relates to the wavevector by $E_{K}=\frac{\hbar^{2} k^{2}}{2 m_{0}}$. A numerical resolution of the previous equation gives access to the band structure of $\mathrm{CdSe}$, the latter is plotted in the inset of Figure 1 (f).

A more intuitive understanding of the band structure is obtained assuming an infinite barrier of potential into the platelets. In this case we can relate the wavevector to the nanoparticle thickness through the relation $k_{N P L}=\frac{\pi}{L_{N P L}}$. Using the band structure parameter previously reported in the literature ${ }^{5}$ and only adjusting the difference between the binding energy of the heavy hole and the light hole we obtain a fair agreement for the energy of the first exciton and heavy hole-light hole splitting, see Figure 1 (g) and (h). In CdSe/CdS the electron band alignment leads to a delocalized electron over the whole structure while the hole remains confined in the CdSe core. Consequently the $\mathrm{HH}-\mathrm{LH}$ splitting is at zeroth order driven by $k_{\text {CdSeCdS }}=\frac{\pi}{L_{\text {core }}}$ for $\mathrm{CdSe} / \mathrm{CdS}$ (i.e. independent of the shell thickness) while for pure CdSe core/shell the impulsion is related to the whole structure thickness by $k_{\text {CdSeCdSe }}=\frac{\pi}{L_{\text {cores/shell }}}$. The fact that the $\mathrm{HH}$-LH splitting vanishes in the CdSe/CdSe structure while it was not the case in the CdSe/CdS NPL confirms that indeed the electron and hole are delocalized over the whole thickness of the NPL. The shell obtained by the method we propose is consequently fully active in the structure confinement. The complete delocalization of the hole wavefunction up to the NPL surface is responsible for the full quench of the PL observed in this material. This behavior significantly differ from the $\mathrm{CdSe} / \mathrm{CdS}$ heterostructure for which the hole confinement is preserved and the PL quantum yield is high. ${ }^{23}$ Ensemble spectroscopic measurements confirm that the growth is equivalent on average to a layer-by-layer procedure. On the other hand at the single particle level, TEM observations tend to show some roughness. In the following we propose to use FET measurements to probe the effect of the growth defect. 


\section{Electrolyte Gating of Thick CdSe Nanoplatelets}

Nanoplatelets, with their large lateral dimensions, are appealing materials for transport since they are good candidates to reduce the number of hopping processes necessary for the carriers to reach the electrodes and thus achieve high effective mobilities. Due to the wide band gap of CdSe, there is little amount of thermally activated carriers, and the film of NPLs has to be gated to obtain reasonable conductance. We recently reported a very efficient gating of a film of nanoparticles ${ }^{19}$ using polymer electrolyte. Our strategy is applicable to a large range of materials and shapes. The good performances obtained are clearly related to the ability to combine an ionic surface chemistry $\left(\mathrm{S}^{2-}, \mathrm{Cd}^{2+}, \mathrm{Se}^{2-}\right.$, i.e. without long non polar capping ligands) with the injection of ions from the electrolyte into the bulk of the film. In spite of this success we were unable to charge a film made of CdSe NPLs core only (even with sulfide capping), which make us wonder if the CdS shell is playing a particular role in the charging process.

We prepare a polymer electrolyte gated FET using the CdSe/CdSe NPLs by dropcasting the NPLs dispersed in NMFA ( $\mathrm{N}$-methyl formamide) on interdigitated electrodes on a hot plate. A mild annealing at $100^{\circ} \mathrm{C}$ is used mostly to eliminate the solvent, while preserving the optical feature. In a second step the soften electrolyte $\left(\mathrm{LiClO}_{4}\right.$ in polyethylene glycol) is brushed directly on the film of particles and a copper grid is used as a gate electrode. Measurements are made, at room temperature, using a probe station. Devices are stable and thus can be operated in air. A scheme of the device is shown on Figure 2 (a).

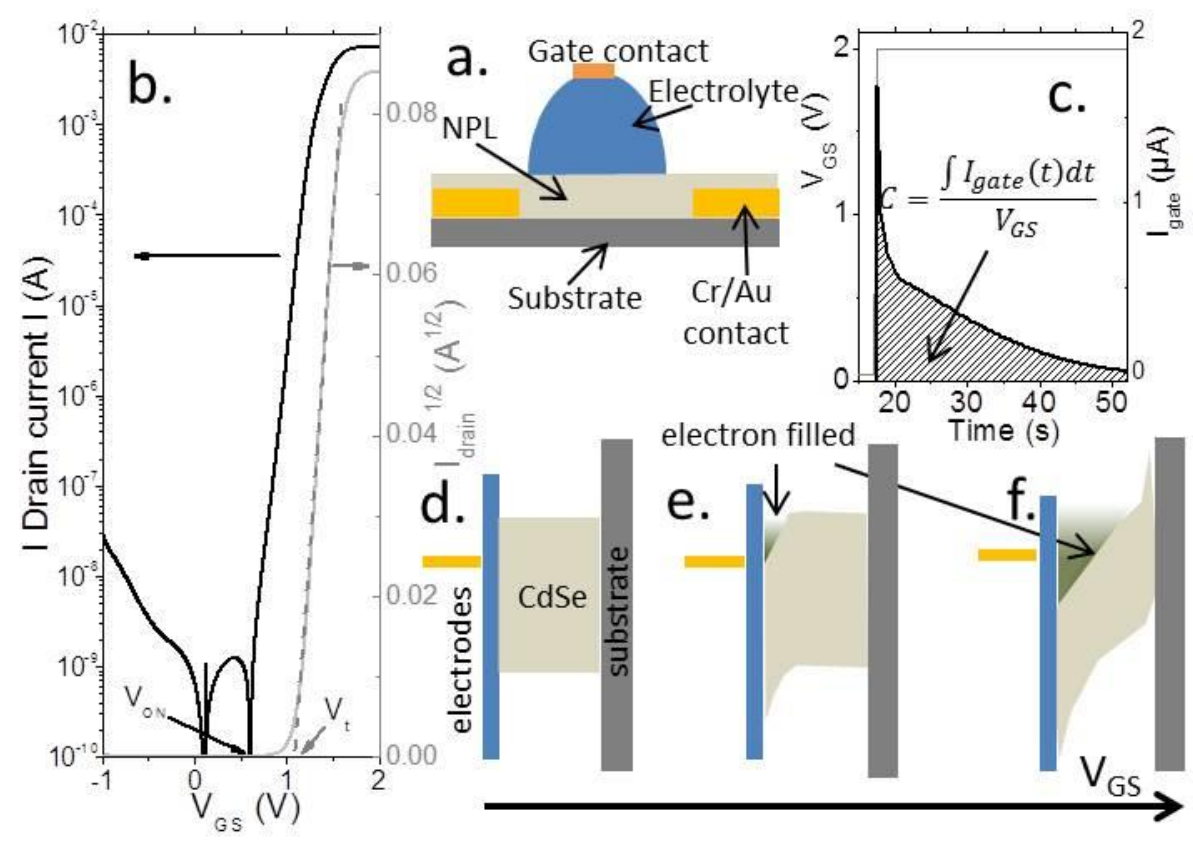

Figure 2 (a) Scheme of the solid electrolyte gated field effect transistor. (b) Drain current as a function of the gate potential for a CdSe/CdSe NPL with $3 M L$ on each side. The drain source bias is set at $0.5 \mathrm{~V}$ and the measurements are made in air at room temperature (c) Time response of the gate current to a $2 \mathrm{~V}$ step of gate bias. The integral of the curve is used to evaluate the capacitance. Schematic band diagram of the electrolyte gated CdSe nanoplatelets film at $V_{G S}=V_{F B}(d)$, at $V_{G S}=V_{t}(e)$ and at $V_{G S}>V_{t}(f)$. 
A strong $\mathrm{n}$-type charging is obtained with low threshold voltage $(\approx 1 \mathrm{~V})$, large on/off ratio (close to $\left.10^{7}\right)$ and small subthreshold swing (100 mV/decade), see Figure 2(b). We evaluate the mobility using the following formula $\left.\mu_{n}^{F E T}=\frac{L}{W C_{\Sigma} V_{D S}} \frac{\partial I_{D S}}{\partial V_{G S}}\right)_{V_{D S}}, L$ is the distance between electrodes (10 or $20 \mu \mathrm{m}$ ), $W$ the electrodes length (typically $25 \times 2.5 \mathrm{~mm}$ ), $C_{\Sigma}$ the surface capacitance evaluated by integrated the gate current and $V_{D S}$ the drain source bias. The electron mobility is estimated to be in the $10^{-2}-10^{-3} \mathrm{~cm}^{2} \mathrm{~V}^{-1} \mathrm{~s}^{-1}$ range. To the best of our knowledge, it is the first time that pure CdSe NPLs are successfully gated using an electrolyte. As a consequence we can conclude that $\mathrm{CdS}$ is not playing a particular role in the gating of CdSe/CdS NPLs. Currently the device is limited in the off state by the leakage through the electrolyte and in the on state by the contact resistance. Lower contact resistance can be obtained by increasing the electrodes thickness. On the other hand it tends to increase the leakage through a direct contact of the electrodes with the electrolyte. The capacitance of the device is high and can be estimated by integrating the gate current in response to a step of gate bias (see Figure 2 (c)). The typical values obtained are in range of the $100-200 \mu \mathrm{Fcm}^{-2}$. Such a large value results from the ability to inject the $\mathrm{Li}^{+}$into the bulk of the film instead of just forming an ionic double layer at the nanoparticle film/electrolyte interface ${ }^{19}$ (see Figure 2 (e) and (f)).

\section{Probing Defects using FET}

We observe that as the CdSe NPLs are grown thicker, the threshold voltage $V_{t}$ (defined as the intercept of the linear fit of $\mathrm{I}^{1 / 2}$ with the zero current axis) and the on/off ratio rise and saturate when 3 CdSe MLs have been added (see Figure 3 (c)). Such an increase of the threshold voltage $V_{t}$ while the bandgap energy of the material is reduced is quite puzzling. Indeed it is generally admitted that $V_{t}$ is correlated with the flat band bias $\left(V_{F B}\right)$ for which the Fermi level of the gate and canal material are aligned $^{24}$ (see Figure 3 (a) and the dashed curve of Figure 3 (c)). According to this idea the threshold voltage should decrease when the band edge energy decreases, i.e. when the NPL thickness increases. Spectroscopically, the CdSe/CdSe NPLs behave as thick uniform CdSe NPLs, and we do not expect that this surprising trend is related to the CdSe material itself. Our hypothesis is that this trend is related to the appearance of defects at the surface of the NPLs, and that the number of defects increases with the shell growth. We tested this hypothesis using electrolyte gated FET since it can probe charge defects on a broad range of density. To understand the threshold dependence with the band edge energy we need to consider the presence of free charges. In this case, the threshold bias can be related to the flat band bias with the relation ${ }^{24} V_{t}=V_{F B}+\frac{Q}{C}$, where $Q$ is the charge involved in the band bending process and, $C$ the capacitance of the electrolyte/nanoparticle interface. $V_{F B}$ can also be related to the band structure parameter via $V_{F B}=\frac{W_{M}-W_{S}}{e}$, where $W_{M}$ and $W_{S}$ are respectively the work function of the metallic gate and of the semiconductor canal, and $e$ is the proton charge. Assuming that for the CdSe/CdSe NPLs, the Fermi energy is roughly in the middle of the gap, the work function of the CdSe/CdSe film is thus equal to the sum of the CdSe electron affinity plus half the band edge energy $\left(E_{B E}\right)$. Given that the copper (used for the gate electrode) has a workfunction ${ }^{25}(4.7 \pm 0.2 \mathrm{eV})$ very close to the bulk CdSe electron affinity ${ }^{26}(4.4 \mathrm{eV}$ - 
$5 \mathrm{eV})$, we can thus estimate the sheet charge involved to reach the threshold voltage by $Q=C\left[V_{t}-\frac{E_{B E}}{2 e}\right]$.

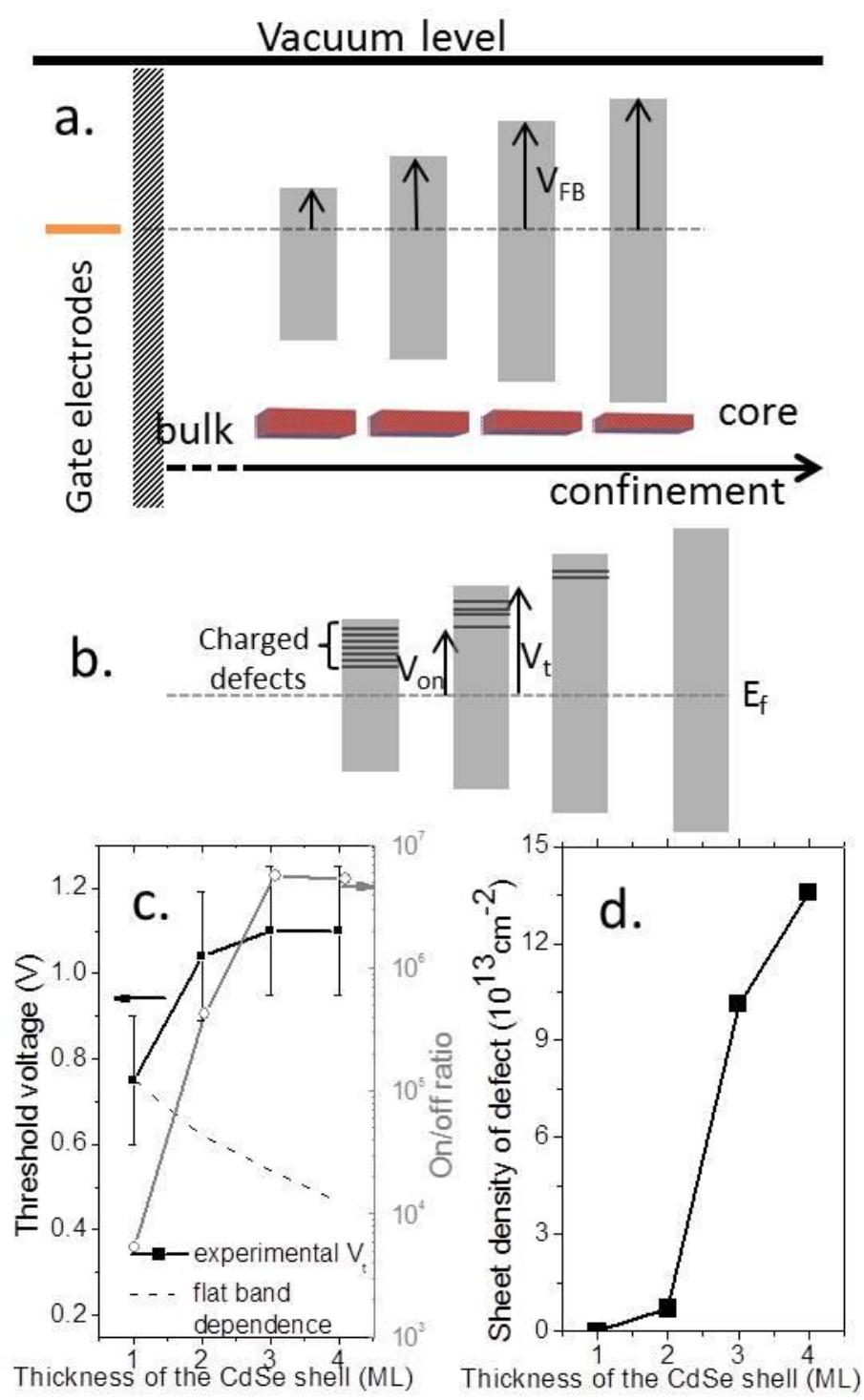

Figure 3 Scheme of the band diagram for CdSe nanoplatelets of different thicknesses without stoichiometry defect (a) and with stoichiometry defects (b). (c) Threshold voltage and on/off ratio as a function of the number of layer in the shell. (d) Sheet density of defects in film of $\mathrm{CdSe} / \mathrm{CdSe}$ as a function of the number of layers in the shell.

While the gate bias rises from the turn-on voltage to the threshold voltage, the charges are typically not injected in the quantum state of the NPLs but instead on trap states ${ }^{27}$ (see Figure 3 (b)). The fact that the associated charge $Q$ rises while the homostructure gets thicker (see Figure 3 (d)), pledges for the formation of defects while the lateral edges of the NPLs get less defined. We believe that such defects are related to stoichiometry defects during the c-ALD growth. This idea is supported by recent result from Oh et al which have confirmed that the c-ALD growth tends to induce some $\mathrm{n}$ or $\mathrm{p}$ 
doping for lead chalcogenides particles. ${ }^{28}$ This density is pretty high several $10^{13} \mathrm{~cm}^{-2}$, or equivalently in the 1 to 10 range of defects per NPL.
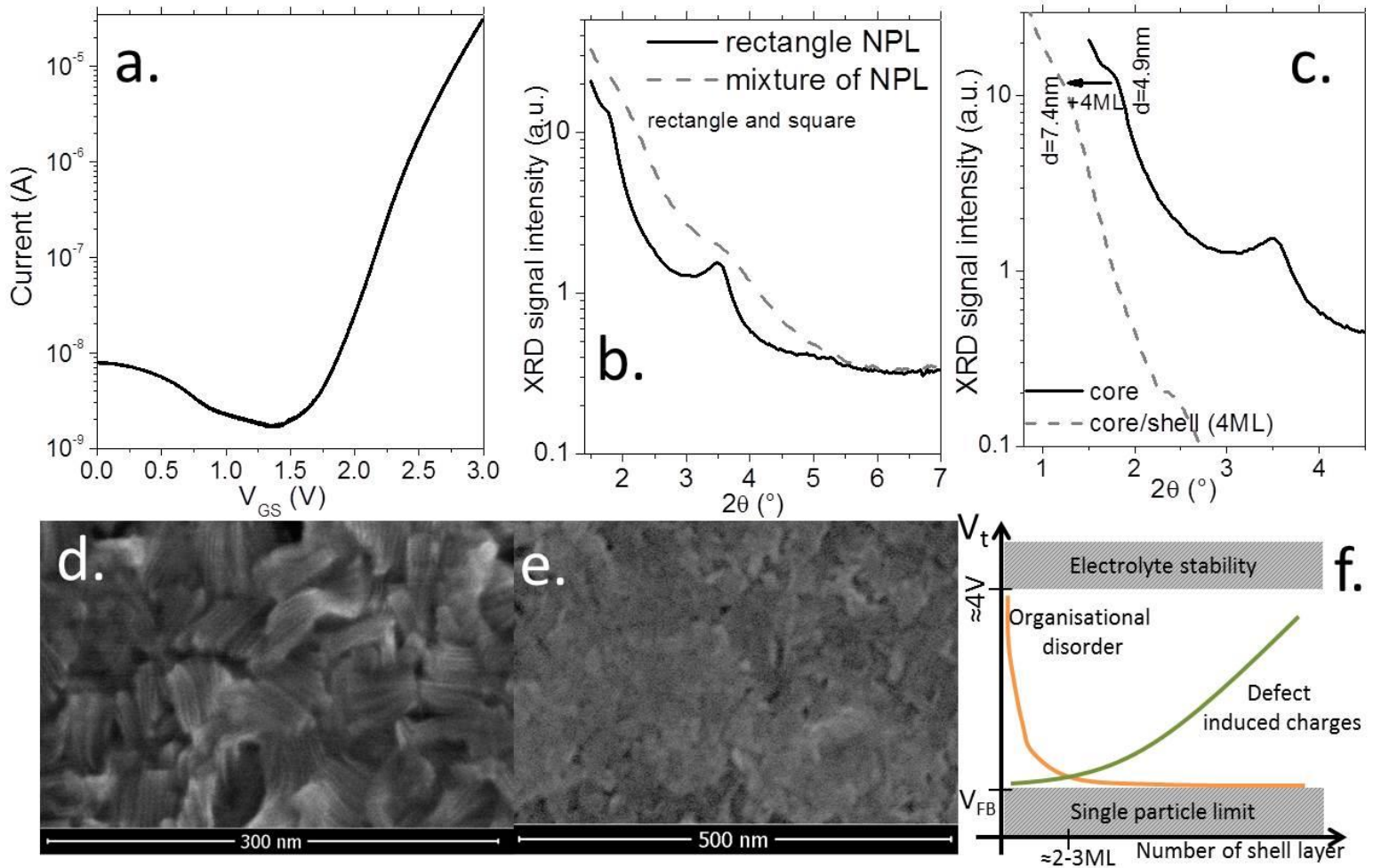

Figure 4(a) Drain current as a function of the gate bias for a solid electrolyte gated field effect transistor based on CdSe core NPLS (mixture of rectangular and square shaped NPL). (b) Low angle X-ray diffractogram of a film of rectangular NPLS and a mixture of rectangular and square shaped NPLs. (c) Low angle X-ray diffractogram of CdSe core NPLs and core/shell (4ML) NPLs. Scanning electron microscopy image of a film of (d) rectangular CdSe NPL (core only) and (e) of a mixture of square and rectangular shape NPL. (f). Scheme of the dependence of the threshold voltage as a function of the number of layers in the nanoplatelets

In the last part of the paper we discuss how the film charging properties are affected by the organization of the nanoparticles at scales larger than the dimensions of the nanoparticles. Our motivation was to understand why in the films made of CdSe core only, NPLs do not get charged, while efficient gating is obtained as soon as a shell (even $1 \mathrm{CdSe} M L$ ) is added. It may have been reasonable to expect a higher charging energy for thinner objects, since the reduction potential scales like the confinement energy. ${ }^{29}$ Consequently a gate bias higher than the stability range of the electrolyte (typically $4 \mathrm{~V}$ ) may have been necessary to charge the core. The CdSe NPL cores we synthetize have extremely monodisperse shapes for both their thickness and lateral extensions, and they tend to stack. ${ }^{30,31}$ These stacks of NPLs are preserved while forming a film, as shown on SEM images in Figure 4 (d) and in Figure S1. The presence of stacked NPLs is also confirmed by the presence of harmonic peaks in the low angle X-ray diffractogram (see Figure 4 (b)). These very dense films are consequently poorly permeable to the diffusion of the $\mathrm{Li}^{+}$cations, and the NPLs films cannot be properly charged. The importance of the pores in a film of nanoparticles regarding its charging 
process has recently been highlighted ${ }^{32}$ To test the importance of the NPLs stacking disorder, we made a film from a mix of CdSe core only NPLs with two different lateral shapes typically rectangles and squares. Neither the square shapes nor the rectangular shape NPL can be charged while processed in a film made of a single shape. For these two kinds of objects the thickness is the same and thus the absorption is the same. Nevertheless the stacking of the NPLs is significantly reduced. The low angle XRD diffractogram is now barely structured (see Figure 4 (b)) and no NPL stack is observed on the SEM image (see Figure 4 (e)). On such a film, n-type charging is successfully achieved (see Figure 4 (a)). However the threshold voltage is higher than the one measured with a thin shell. Films of NPL with even less long range order will very likely leads to a stronger charging thanks to higher ionic permeability.

From this result we can conclude that charging of the CdSe NPLs can be obtained as long as the film includes enough voids to let the ions diffuse. Thanks to this result we can also reconsider the effect of the CdSe shell. The shell introduces some defects in the stacking of the NPLs, due to the thickness and lateral extension roughness. Indeed as for mixture of NPLs with different shape we observe a less structured low angle diffraction pattern (see Figure 4 (c)). Figure 4 (f) summarizes the dependence of the threshold voltage with organizational disorder and stoichiometry defects. In absence of structural disorder, $V_{t}$ is high due to the difficulty to let the electrolyte ions percolate in the nanoparticles film and more gate bias is necessary to induce the ions displacement into the nanoparticles film. When disorder is introduced, $V_{t}$ quickly drops and the $\mathrm{Li}^{+}$cations are allowed to explore more percolation paths in the nanoparticle film. Moreover $V_{t}$ is strongly dependent on the absolute energy band diagram which is itself affected by the presence of stoichiometry defects.

\section{CONCLUSION}

We propose a method to quantify the amount of stoichiometry defects in colloidal nanoparticle using simple polymer electrolyte gating. This method is applied to probe the growth defects induced by a CdSe shell grown on CdSe NPLs for which spectroscopy confirms the delocalization of both the electron and holes. The use of this material in a solid electrolyte gated FET leads to FET with remarkable properties, such as turn on voltage as small as $0.5 \mathrm{~V}$, large on /off ratio (close to $10^{7}$ ) and small subthreshold swing slope ( $100 \mathrm{mV} /$ decade). We demonstrate that the NPL organization needs to be loose enough to let the ions percolate through the whole film. Such control can be obtained either by tuning the surface of the particle (shell growth) or by controlling the nanoparticle organization.

We can conclude that the shell role is double (i) first it reduces the NPL stacking, probably due to a limited surface inhomogeneity, which allows the cations to diffuse into the film and form a ionic double layer around the NPLs. (ii) Secondly the shell growth leads to some stoichiometry defects and traps which have to be filled before injecting the charges on the quantum states. This study also confirms that optical spectroscopy and field effect measurement are two complementary tools to probe the full band structure including quantum and trap states of colloidal nano-objects. 


\section{METHOD}

\section{Materials}

n-Hexane (VWR, 98\%), ethanol (VWR, 98\%), methanol (VWR, 100\%), N-methyl formamide (NMFA) (VWR, 99\%), acetonitrile (Merck, 99.8\%), toluene (Sigma Aldrich, 99.8\% anhydrous), Se powder (Sigma-Aldrich, 99.99\%), cadmium nitrate tetrahydrate (Strem, 98\%), Cadmium acetate dihydrate $\left(\mathrm{Cd}(\mathrm{OAc})_{2} .2 \mathrm{H}_{2} \mathrm{O}\right)$ (Aldrich $\left.98 \%\right)$, Oleic acid (Aldrich, 90\%), $\mathrm{Na}_{2} \mathrm{~S} .9 \mathrm{H}_{2} \mathrm{O}$ (Sigma-Aldrich, 99.5\%), myristic acid (Sigma, 99\%), $\mathrm{LiClO}_{4}$ (Aldrich, 98\%), polyethylene glycol $\mathrm{Mw}=6 \times 10^{3} \mathrm{~g} \cdot \mathrm{mol}^{-1}$ (PEG 6k)(Fluka), $\mathrm{NaBH}_{4}$ (Sigma Aldrich, 98\%), $\mathrm{NaOH}$ (Aldrich, 98\%). All precursors are used as received. All the solvents are degassed by bubbling with argon and then dried on $4 \AA$ molecular sieves.

\section{Synthesis of the CdSe Nanoplatelets Core Absorbing at 510nm}

The CdSe nanoplatelets are synthetized using the procedure previously described ${ }^{2}$. Briefly in a $100 \mathrm{~mL}$ three neck flask $340 \mathrm{mg}(0.6 \mathrm{mmol})$ of cadmium myristate are introduced along with $23.4 \mathrm{mg}(0.3 \mathrm{mmol})$ of Se powder and $30 \mathrm{~mL}$ of ODE. The solution is degased under vacuum for $30 \mathrm{~min}$ at room temperature. Under $\mathrm{Ar}$ flow, the temperature is set at $240^{\circ} \mathrm{C}$. When the temperature reaches $200^{\circ} \mathrm{C}$ (the solution is yellow orange at this step), $80 \mathrm{mg}(0.3 \mathrm{mmol})$ of cadmium acetate are quickly added to the solution. Finally the reaction is left for $12 \mathrm{~min}$ at $240^{\circ} \mathrm{C}$. $2 \mathrm{~mL}$ of oleic acid are then injected to quench the reaction and the flask is cooled down to room temperature. The nanoparticles are precipitated by adding 1.5 volume equivalent of ethanol. After centrifugation at $4000 \mathrm{rpm}$ for 10 minutes, the clear supernatant is discarded and the precipitated pellet is redisolved in fresh hexane $(8 \mathrm{~mL})$. The cleaning procedure is repeated a second time.

\section{Synthesis of the CdSe Shell}

In the glove box, a Se solution precursor is prepared by dissolving Se powder $(0.3 \mathrm{mmol})$ with two equivalents of $\mathrm{NaBH}_{4}(22.7 \mathrm{mg}, 0.6 \mathrm{mmol})$ in a $1.5 \mathrm{ml}$ mixture of NMFA and ethanol (50:50 as volume ratio). To avoid a too strong reduction of the hydride, the precursor is slowly introduced. The solution switches from dark to clear after complete reduction of the selenium. The final solution is filtered using $0.2 \mu \mathrm{m}$ filter.

Inside the glove box the Cd precursor is obtained by disolving cadmium acetate in NMFA. To grow the shell on the NPL, $1 \mathrm{~mL}$ of the NPL core is mixed with $1 \mathrm{~mL}$ of NMFA, then $80 \mu \mathrm{L}$ of the Se solution is introduced in the NPL solution. After strong mixing the solution turns redder. The non polar phase is washed by adding hexane. When the obtained non polar phase is clear, this phase is discarded. The particles are precipitated by adding a 3:1 volume mixture of toluene and acetonitrile. After centrifugation the clear supernantant is discarded and the pellet is dispersed in $0.75 \mathrm{~mL}$ of fresh NMFA. Then $80 \mu \mathrm{L}$ of the $\mathrm{Cd}$ precursor solution is added. After strong mixing the solution is precipitated by adding the same toluene acetronitrile mixture. Once centrifuged the particles are redispersed in NMFA. This process is repeated up to obtain the desired number of CdSe layers. The absorption spectra and microscopy image are shown on Figure 1 . They have a typical size of $10 \mathrm{~nm} \times 20 \mathrm{~nm}$.

\section{Polymer Electrolyte Gated Field Effect Transistor}

Interdigitated electrodes (25 pairs with a 10 or $20 \mu \mathrm{m}$ spacing, each digit is $2.5 \mathrm{~mm}$ long and $10 \mu \mathrm{m}$ wide) of $\mathrm{Cr}$ $(3 \mathrm{~nm})$ gold $(30 \mathrm{~nm})$ are prepared by standard optical lithography and thermal evaporation methods. The nanoparticles dispersed in NMFA are dropcasted on the electrodes on a warm hot plate $\left(100^{\circ} \mathrm{C}\right)$. The electrolyte is obtained by melting $50 \mathrm{mg}$ of $\mathrm{LiClO}_{4}$ in $230 \mathrm{mg}$ of PEG at $165^{\circ} \mathrm{C}$ on a hot plate in the glove box for 90 minutes. The final solution is homogeneous and can easily be brushed on the nanoparticles film.

\section{Material and Electrical Characterization}

UV-Visible absorption spectra are measured using a Cary $5 \mathrm{E}$ spectrometer. Electrical measurements are acquired in air with two Keithley 2400 sourcemeter. The gate bias scan rate is typically $1 \mathrm{mV}^{-1}{ }^{-1}$. Transmission electron microscopy (TEM) image were acquired at $200 \mathrm{keV}$ with a JEOL JEM 2200FS scanning transmission electron microscope equipped with a CEOS aberration-corrector. X-ray diffraction was measured on a X'pert 
Philipps system using the copper Ka line. Energy dispersive X-ray spectroscopy (EDX) is obtained on an Inca energy Oxford instrument system.

\section{ACKNOWLEDGEMENTS}

We are grateful to $X$. Xu for performing the TEM measurements. We thank H. Aubin for fruitful discussions. BD thanks the ANR agency for funding.

\section{SUPPLEMENTARY MATERIALS}

Additional information of the precursor preparation, synthesis of square shapes NPLs, dark field TEM images, wide angle X-ray diffraction data are given in the additional material. This material is available free of charge via the Internet at http://pubs.acs.org.

\section{REFERENCES}

${ }^{1}$ Peng, X. G.; Manna, L.; Yang, W. D.; Wickham, J.; Scher, E.; Kadavanich, A.; Alivisatos, A. P., Shape Control of CdSe Nanocrystals, Nature 2000, 404, 59-61.

2 Ithurria, S.; Dubertret, B. Quasi 2D Colloidal CdSe Platelets with Thicknesses Controlled at the Atomic Level, J. Am. Chem. Soc. 2008, 130, 16504-16505.

3 Joo J.; Son J.S.; Kwon S.G., Yu J.H.; Hyeon T. Low-Temperature Solution-Phase Synthesis of Quantum Well Structured CdSe Nanoribbons, J. Am. Chem. Soc., 2006, 128, 5632-5633.

4 Tessier, M. D.; Javaux, C.; Maksimovic, I.; Loriette, V.; Dubertret, B., Spectroscopy of Single CdSe Nanoplatelets. ACS Nano 2012, 6, 6751-6758.

${ }^{5}$ Ithurria, S.; Tessier, M. D.; Mahler, B.; Lobo, R. P. S. M.; Dubertret, B.; Efros, A. L. Colloidal nanoplatelets with Two-Dimensional Electronic Structure. Nat. Mater. 2011, 10, 936-941.

${ }^{6}$ Li, Z.; Qin, H. Y.; Guzun, D.; Benamara, M.; Salamo, G.; Peng, X. G. Uniform Thickness and Colloidal-Stable CdS Quantum Disks with Tunable Thickness: Synthesis and Properties. Nano Res. 2012, 5, 337-351.

7 Li Z.; Peng X. Size/Shape-Controlled Synthesis of Colloidal CdSe Quantum Disks: Ligand and Temperature Effects J. Am. Chem. Soc., 2011, 133, 6578-6586.

8 Pedetti S.; Nadal B. ; Lhuillier E. ; Mahler B.; Bouet C. ; Abécassis B. ; Xu X. ; Dubertret B. Optimized Synthesis of CdTe Nanoplatelets and Photoresponse of CdTe Nanoplatelets Films, Chem. Mat 2013, 25, 2455-2462.

9 Liu Y.H.; Wayman V.L.; Gibbons P.C., Loomis R.A.; Buhro W.E., Origin of High Photoluminescence Efficiencies in CdSe Quantum Belts, Nano Lett. 2010, 10, 352-357

10 Mahler B.; Nadal B.; Bouet C.; Patriarche G.; Dubertret B. Core/Shell Colloidal Semiconductor Nanoplatelets, J. Am. Chem. Soc. 2012, 134, 18591-18598.

11 Ithurria, S.; Talapin, D. V. Colloidal Atomic Layer Deposition (c-ALD) Using Self-Limiting Reactions at Nanocrystal Surface Coupled to Phase Transfer between Polar and Nonpolar Media, J. Am. Chem. Soc. 2012, 134, 18585-18590

12 Talapin, D. V., Lee, J. S., Kovalenko, M. V. \& Shevchenko, E. V. Prospects of Colloidal Nanocrystals for Electronic and Optoelectronic Applications, Chem. Rev. 2010, 110, 389-458.

${ }^{13}$ Wanger D.D., Correa R.E., Dauler E.A., Bawendi M.G. The Dominant Role of Exciton Quenching in PbS Quantum-Dot-Based Photovoltaic Devices, Nano lett asap (2013)

${ }^{14}$ Kang, M. S., Lee, J., Norris, D. J., Frisbie, C. D. High Carrier Densities Achieved at Low Voltages in Ambipolar PbSe Nanocrystal Thin-Film Transistors. Nano Lett. 2009, 9, 3848-3852.

15 Bisri S.Z., Piliego C., Yarema M., Heiss W., Loi M.A., Low Driving Voltage and High Mobility Ambipolar Field-Effect Transistors with PbS Colloidal Nanocrystals. Adv Mat 2013, 25, 4309-4314. 
${ }^{16}$ Yu D., Wang C., Guyot-Sionnest P., N-type Conducting CdSe Nanocrystal Solids, Science 2003, 300, $1277-$ 1280

${ }^{17}$ Vanmaekelbergh, D., Houtepen, A. J. \& Kelly, J. J. Electrochemical Gating: a Method to Tune and Monitor the (Opto)Electronic Properties of Functional Materials, Electrochim. Acta 2007, 53, 1140-1149.

18 Vanmaekelbergh D., Liljeroth P. Electron-Conducting Quantum Dot Solids: Novel Materials Based on Colloidal Semiconductor Nanocrystals, Chem. Soc. Rev. 2005, 34, 299-312

19 Lhuillier E.; Aubin H.; Ithurria S.; Dubertret B. A versatile Method to Build Metal Chalcogenides Nanoparticles Based Electrolytic Transistor, submitted 2014.

${ }^{20}$ Nag A.; Kovalenko M.V.; Lee J.S.; Liu W.; Spokoyny N.; Talapin D.V. Metal-free Inorganic Ligands for Colloidal Nanocrystals: S2-, HS-, Se2-, HSe-, Te2-, HTe-, TeS32-, OH-, and NH2- as Surface Ligands. J Am Chem Soc 2011, 133, 10612-10620.

${ }^{21}$ Klayman D. L ., Griffin T. S., Reaction of Selenium with Sodium Borohydride in Protic Solvents. A Facile Method for the Introduction of Selenium into Organic Molecules, J. Am. Chem. Soc. 1973, 95, 197-199.

${ }^{22}$ Fishman G. "Semi-conducteurs : les bases de la therorie kp », Les éditions de l'école polytechnique, first edition, 2010.

${ }^{23}$ Tessier, M. D.; Mahler, B.; Nadal, B.; Pedetti, S.; Heuclin, H., Dubertret, B., Spectroscopy of Colloidal Semiconductor Core/Shell Nanoplatelets with High Quantum Yield. Nano Letters 2013, 13, 3321-3328.

${ }^{24}$ Kergoat L.; Herlogsson L.; Piro B.; Pham M.C.; Horowitz G.; Crispin X.; Berggren M. Tuning the Threshold Voltage in Electrolyte-Gated Organic Field-Effect Transistors Proc. Nat. AC. Soc. 2012, 109, 8394-8399.

${ }^{25}$ Li W.; Li D.Y. On the Correlation between Surface Roughness and Work Function in Copper, J. Chem. Phys. 2005, 122, 064708.

${ }^{26}$ Nethercot A.H. Prediction of Fermi Energies and Photoelectric Thresholds Based on Electronegativity Concepts, Phys Rev. Lett. 1974, 33, 1088-1091.

${ }^{27}$ Stadler P.; Sutherland B.R.; Ren Y.; Ning Z.; Simchi A.; Thon S.M.; Hoogland S.; Sargent E.H. Joint Mapping of Mobility and Trap Density in Colloidal Quantum Dot Solids, ACS Nano 2013, 7, 5757-5762.

${ }^{28}$ Oh S.J., Berry N.E., Choi J.H., Gaulding E.H. , Lin H., Paik T., Diroll B. T. , Muramoto S., Murray C.B., Kagan C. R., Designing High-Performance $\mathrm{PbS}$ and PbSe Nanocrystal Electronic Devices through Stepwise, PostSynthesis, Colloidal Atomic Layer Deposition, Nano Lett. asap (2014)

${ }^{29}$ Wang C.; Shim M.; Guyot-Sionnest P. Electrochromic Nanocrystal Quantum Dots, Science 2001, 291, $2390-$ 2392.

${ }^{30}$ Tessier M.D.; Biadala L.; Bouet C.; Ithurria S.; Abecassis B.;Dubertret B. Phonon Line Emission Revealed by Self-Assembly of Colloidal Nanoplatelets, ACS Nano, 2013, 7, 3332-3340

${ }^{31}$ Abécassis B., Tessier M.D., Davidson P., Dubertret D., Self-Assembly of CdSe Nanoplatelets into Giant Micrometer-Scale Needles Emitting Polarized Light, Nano Lett asap (2013)

${ }^{32}$ Boehme S.C.; Wang H.; Siebbeles L.D.A.;Vanmaekelbergh D.; Houtepen A.J. Electrochemical Charging of CdSe Quantum Dot Films: Dependence on Void Size and Counterion Proximity, ACS Nano, 2013, 7, 25002508 